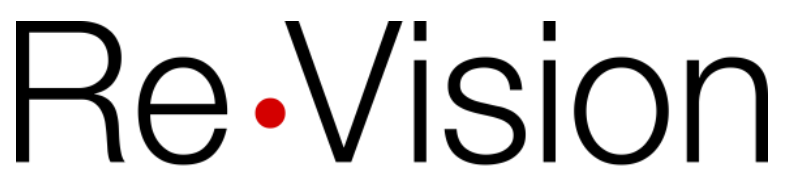

UNIVERSITY

ofGUELPH

The Centre for Art and Social Justice

The Eating Disorder Recovery

Assemblage: Collectively

Generating Possibilities for Eating

Disorder Recovery

Andrea LaMarre

Massey University

Carla Rice

University of Guelph

This is a post-peer-review, pre-copyedit version of an article published in Feminism \& Psychology. The final authenticated version is available online at: https://doi.org/10.1177/0959353520941353

Recommended citation:

LaMarre, A., \& Rice, C. (2020). Affecting recovery: Collectively generating possibilities for eating disorder recovery. Feminism \& Psychology. https://doi.org/10.1177/0959353520941353 


\section{The Eating Disorder Recovery Assemblage: Collectively Generating Possibilities for Eating Disorder Recovery}

Andrea LaMarre \& Carla Rice

* Note: work carried out while Andrea LaMarre was affiliated with the University of
Guelph

\section{Abstract}

In this paper, we explore the affective-discursive-material aspects of the supportive eating disorder recovery assemblage. We approach recovery as an "assemblage" to facilitate an understanding of how human (people, systems of care, etc.) and nonhuman (affect, discourses, etc.) forces generate possibilities or impossibilities for recovery. Moving away from framings of recovery as an individual achievement, we consider the relationality and dynamism of eating disorder recovery in interviews with 20 people in recovery and 14 supporters of people in recovery. We draw from experiential accounts to theorize a supportive eating disorder recovery assemblage in relation to trust and love mobilized in interactions and relationships. This supportive eating disorder recovery assemblage can scaffold new understandings of recoveries as multiple and co-produced. Supportive eating disorder recovery assemblages generate improvisational spaces, albeit loosely contained and bounded, for different pathways to and manifestations of "recoveries". This work builds on a body of feminist scholarship on eating disorders/disorder eating that takes up gendered 
relationships of power in treatment settings, extending toward and analysing material, affective, embodied, and potentially affirming dimensions of care and emotion in participants' lives.

\section{Key Words}

Affect, emotion, eating disorders, recovery, qualitative, Canada

\section{Acknowledgments}

Parts of this manuscript were a part of the first author's dissertation and is online via institutional repository (The Atrium, University of Guelph). Parts of this manuscript were presented verbally at the Psychology of Women and Equalities Section Conference of the British Psychological Society in 2018. We also wish to acknowledge participants in recovery and supporters for sharing their stories. We thank the eating disorder scholars and activists whose work has inspired us and continues to drive work toward systems change in the hopes that more people will one day feel safe enough to share their stories.

\section{Funding Acknowledgment}

The first author's dissertation research was generously funded by the Vanier Canada Doctoral Scholarship (CIHR) and subsequently by the Ontario Ministry for Longterm Care through the Ontario Women's Health Scholarship. The views expressed in the material are ours and do not necessarily reflect those of the Ministry of Health and Long-Term Care. 
"Eating disorders" 1 and recovery are popularly considered to be issues of individual concern, despite feminist scholars' analyses of the intersection between disordered eating and societal standards for performances of femininity and masculinity (e.g. Bordo, 1993; Gremillion, 2003; Malson \& Burns, 2009). Existing research on recovery considers how social networks can provide support to those experiencing distress around food and in their bodies (e.g. Linville, Brown, Sturm \& McDougal, 2012) and delineates the burdens that supporters may face (e.g. Whitney, Haigh, Weinman \& Treasure, 2007). However, the ultimate onus of recovery is still often placed on the person in recovery. Suggestions that "a person cannot get better until they want to" and that "you need to choose recovery" carry forceful assertions: that recovery inheres within the individual, who has (or lacks) the capacity/will to make autonomous choices that scaffold a recovered life. This construction may close out complex understandings of the interconnected aspects of life that make recovery possible (or impossible) for different people in different places, times, and relational configurations.

Deeply implicating bodies, contexts, discourses and other forces that give shape to everyday life in analyses of eating disorder recovery opens up possibilities for reenvisioning who and what is involved in creating space for recoveries. Supporters of

\footnotetext{
${ }^{1}$ We recognize the potentially problematic nature of the use of "eating disorder" as a terminology to refer to people's lived experiences of distress around food and in their bodies. In this paper, we use this term as it is one that resonated with many of our participants, but it also cannot be abstracted from its pathological legacy and exclusions. We default to participants' preferences as an acknowledgment of their lived experiences as they express them.
} 
people working to establish a sense of greater ease in their bodies and around food after a period of distress are themselves tied up in assumptions about what it means to be "healthy" in contemporary Western contexts. And yet, they are often configured as "resources" for recovery without a deep exploration of how they might do the work of enabling recoveries. As feminist affect theorist Sara Ahmed writes, "we are moved after all by the proximity of others. We feel with and for others" $(2008, p .10)$. In order to explore supportive eating disorder recovery relationships in a nuanced, embodied, and situated way, it is necessary to think about relationships differently - as more than simply acting on or being a resource for another.

Critical feminist work on eating disorders has explored the intimate and inextricable linkages between power and care in treatment settings (Lester, 2018). There are "material and affective realities" (p. 517) that surround care (and the withholding of care and responsiveness) and complicate questions of agency and choice in the treatment context. For example, conditions of care (including managed care, clinical and theoretical orientations to eating disorders, tensions around program design, and more) set the stage for particularized framings of behaviour that generate no-win situations for people with eating disorders (Lester, 2019). Explorations that attend to the power and relational dynamics of treatment and recoveries from anthropologists (e.g. Eli, 2014; Gremillion, 2003; Lavis, 2015; Lester, 2019), cultural studies scholars (e.g. Bordo, 1993), and psychology researchers (e.g. Malson, 1998; Malson \& Burns, 2009) have meaningfully expanded the lens on what eating disorders are and how they are treated. We take up this orientation to eating disorders as 
contextually situated and always tied to power and relationships and focus specifically on recoveries.

In this article, we focus on the various forces that impact on and flow through experiences of eating disorder recovery to theorize the supportive eating disorder recovery assemblage. We explore these in relation to the mobilization of trust and love within a biopedagogical context. In so doing, we approach eating disorder recovery as an assemblage (Deleuze \& Guattari, 1987). We understand assemblage, here, as a provisional arrangement made up of people and material things (treatment providers, food, supports, etc.) but also immaterial aspects, including "relational connections and affective forces and agential capacities" (Lupton, 2019, p. 2002). To explore this assemblage, we bring a new materialist theoretical lens to our analysis. We are inspired by the work of other recovery scholars across disciplines and topic areas, including Duff $(2014,2016)$ who theorizes recovery as a "collective, relational achievement" (Duff, 2016, p. 70). Bringing such perspectives to bear on eating disorder recovery means attending to relational, entangled, affective, and dynamic behaviours and ways of being —on the part of people in recovery, but also their supporters and others in their social worlds.

\section{Thinking with Theory Through "Eating Disorder Recovery Assemblages"}

There are robust examples of quantitative and qualitative studies on eating disorder recovery that share particularized accounts of what eating disorder recovery "is" and how to get there. In general, these accounts gesture at the need to see recovery as more than simply remitting from symptoms, for example by exploring 
psychosocial recoveries and quality of life (Bardone-Cone et al., 2018; de Vos et al., 2017). Increasingly, researchers are lucid about how categorization systems for eating disorders and recovery do not capture the grey-area between "illness" and "wellness" in recovery. For instance, Slof'opt-Landt et al. (2019) reflect on how those in recovery may experience a higher quality of life despite lower scores on standardized questionnaires, suggesting that happiness can co-exist with emotional struggle/distress. Likewise, Romano \& Ebener (2019), in their exploration of perceptions of wellbeing amongst people in recovery, deem these "idiosyncratic" and argue for the need to integrate lived experiences into definitions of recovery. Qualitative analyses have often positioned recovery as a process of "becoming whole" (Jenkins \& Ogden, 2012) or as a series of "turning points" (Matoff \& Matoff, 2001). These analyses, while often providing rich accounts of recoveries and their varied sociocultural textures, tend to focus on recovery as individual achievement with a specific, predetermined endpoint.

Expanding out beyond the boundaries of the individual recovering subject might allow us to imagine ways forward that open to different types of recoveries. Envisioning recovery as an "assemblage" (Deleuze \& Guattari, 1987) allows us to think about the various human and non-human forces that generate possibilities or impossibilities for recovery. This represents a radical shift away from presenting recoveries as "survival of the fittest," gritted-teeth trudges through the sociocultural mud of contemporary Western cultures. Since the 1990s, feminist work on eating disorders has compellingly unpacked the sociocultural discourses that constrain all people, including those with eating disorders (e.g. Bordo, 1993). Bringing this lens to recovery, we might explore the multiplicity of forces that comprise recovery (Duff, 2014, 2016; Price-Robertson, 
Manderson \& Duff, 2017). Recovery assemblages are comprised of "the messy constellation of people, institutions, environments and objects on the whiteboard" (PriceRobertson et al., 2017, p. 2). In other words, recovery assemblages are multiple, interwoven, and always situated within relational, affective, and sociocultural contexts.

\section{Biopedagogies}

When we aim to explore this "messy constellation" of recoveries (PriceRobertson et al., 2017, p. 2) it is important to consider how people who make up the eating disorder support assemblage are themselves bound up in dominant cultural discourses on what it means to be "healthy" or "well." To do this, we turn to an exploration of biopedagogies or teachings about health and wellness that "manage" bodies and form a part of surveillance apparatuses (Wright \& Harwood, 2009), circulating around and through individual bodies in targeted populations as "technologies of the self" (Foucault, 1988).

Biopedagogies have been used to understand the challenge of prevention of (LaMarre, Rice \& Jankowski, 2017) and recovery from eating distress (LaMarre \& Rice, 2016; Rinaldi, LaMarre \& Rice, 2016), mothering and food (Cappellini, Harman \&

Parsons, 2018), the "obesity epidemic" and attempts at its "control" (Wright \& Harwood, 2009; Rice, 2014, 2015), and health promotion in general, amongst other topics where people are expected to make choices about health and their bodies to support their becoming healthy productive citizens (e.g., eat less, control weight, etc.). Biopedagogies help us to understand how bodies and "choices" about body management are entangled in flows and affects, structural conditions, and 
understandings of what it means to be a) a person labelled with an eating disorder and b) a "normal" healthy citizen in a world that frames health in arguably problematic and certainly very specific and exclusionary terms (LaMarre \& Rice, 2016; Rice, 2014; Rail \& Jette, 2015).

Understanding biopedagogies can help us to explore lived experiences of recovering into a culture that holds distinctive imperatives for bodies and health. They can also help us to configure prospective supporters as themselves entangled in their own socially contextualized relationships with food, eating, bodies, exercise. Given that biopedagogies exert a subtle and diffuse power throughout multiple-arguably allspheres of life (MacNeill \& Rail, 2010), it is important to also consider the information and dictates supporters might navigate as they work to support people in recovery. Biopedagogies also surround parenting (Burrows, 2009; Wright \& Halse, 2013), partnering, and, potentially, expectations to surveil and control those experiencing eating distress issued by authorities and experts on eating disorders treatment.

As a part of eating disorder recovery assemblages, supporters may come into confrontation with the ways their own eating, exercise, and other embodied experiences replicate and/or challenge dominant perspectives on what "health" means. Discontent in bodies has become normative in contemporary Western societies (Rodin, Silberstein \& Streigel-Moore, 1984; Tantleff-Dunn, Barnes \& Larose, 2011). Body image promotion work has explored how a first step toward preventing discontent might be for those in a teaching position to explore their own body bias and internalization of anti-fat bias (Piran, 2004). Yet, less work has interrogated the experiences of supporters living in a world wherein negative body image is a facet of normative embodied lives and as they 
observe/participate in eating disorder recovery. Navigating multiple and conflicting biopedagogies may be confusing (Cliff \& Wright, 2010; LaMarre, Rice \& Jankowski, 2017) and may generate varied and contradictory affective fields that render supporters feeling alternately empowered and stuck. Exploring experiences at the interstice of dominant and eating disorder biopedagogies (LaMarre \& Rice, 2016) may reveal new insight not only into embodied recoveries, but also social, relational, and affective ones.

\section{Affect}

Theorizing affect in the social sciences can be a somewhat ephemeral task, risking under-articulated explorations of the "uncanny" that leave the "what" of affect unclear (Wetherall, 2012). We came to this work on eating disorder recovery assemblages with a desire to put words to the uncanny - the "reasons" for which some relationships were described as helpful, why certain people were selected for inclusion in the broader study, and what aspects of a supportive recovery relationship makes possible new ways of "being recovered." As we conducted our analysis and throughout the writing process, we wrestled with affect. We began with an exploration grounded in Massumi (2015), Braidotti (2002), and Clough's (2003) articulations of affect. These scholars theorize the "lines of flight" (Deleuze \& Guattari, 1987) or flows and workings of affect, untethering affect from its humanist leanings (i.e. that affects are a largely troublesome part of human experience, are shared across cultures, emerge from within, and are categorizable as good or bad) and theorizing a playful and improvisational affect that is embodied and felt but never truly knowable. 
In this vein, Massumi (2015) looks at how affect "flips over" relations and emerges in bursts-affect, here, is not emanating from one particular person or even necessarily perceivable at a conscious level. Braidotti (2002) orients to affect as hopeful and productive force-in other words, she looks at the transformative capacity of affect. In turn, Clough (2003) brings affect into analyses of political and colonizing forces, for instance unpacking affect as a subtle and visceral "net" through which people and their bodies are pacified through the "modulation of moods, capacities, affects, potentialities" (p. 360). For Clough, we might make sense of both biopedagogies and affect as entwined modes of governance under heteropatriarchal neoliberal capitalism.

We also became attuned to Sara Ahmed's articulation of affect. For Ahmed (2004), emotion as affective force circulates and transmits in and across bodies in ways that bind them together and define them against other bodies or things; it functions like an economy wherein different currencies of emotion are distributed and invested (Ahmed, 2004). Affect, here, is necessarily relational—even intercorporeal—as it operates in between bodies. Attending to affect helps us to analyze the glue that binds existing bonds and to envision new possible relational configurations (Gregg \& Seigworth, 2010; Stephens, 2015).

In terms of practically orienting to affect in analysis, there is the risk that in such non-representational approaches to theorizing affect, the contextual, historical, and discursive might be lost (Wetherall, 2012). Affect operates between people, rather than inhering within people (Ahmed, 2004). And yet, to explore the affective aspects of supportive eating disorder recovery relationships, we also feel drawn to an approach that allows us to "locate affect, not in the ether, or in endless and mysterious 
circulations, but in actual bodies and social actors, negotiating, making decisions, evaluating, communicating, inferring and relating" (Wetherall, 2012, p. 159).

As researchers invested in an approach to research that implicates our own embodiment in our research practices (Rice 2009; 2018), we felt the operation of affect in the supportive eating disorder recovery assemblages as they were described. Affect leaked into the interview space in pauses, tears, and gestures-our own, and those of participants. And yet, exploring affect retrospectively and in the interview transcripts analyzed carried only pale shadows of the visceral eruption of affect that occurred in the moment of the interview as participants described recovery and the relationships and practices that sustained this for them. In order to not go beyond the scope of the data we interpret in this piece, we conceive of affect as one aspect of the eating disorder recovery assemblage; a part of the weaved-together and dynamic bringing together of human and non-human forces (Lupton, 2019). We understand recovery to be collectively assembled and brought together through embodiment, affect, and context (Duff, 2016; Fullagar, 2020; Price-Robertson, Manderson \& Duff, 2017). In future research, we aim to continue to investigate the operation of affect in eating disorder recovery assemblages.

\section{Methods}

\section{Theory and Methodological Approach}

In the broader study from which this work is drawn, we took a new materialist, feminist approach to reflexive thematic analysis (Braun \& Clarke, 2006, 2019). We attended to themes at the latent level, looking at the data in relation to broader social 
meaning and contexts. For the larger study, reflexive thematic analysis was a helpful methodological approach as it allowed us to integrate theory into our analyses and to position ourselves as active in the analysis (Braun \& Clarke, 2019). Perhaps most importantly, using thematic analysis allowed us to identify patterns in the conceptualization of "recovery" from various interviewees that broadened beyond the often psychometrically oriented or pre-defined approaches to measuring and understanding recovery. In this paper, we theorize one aspect of a broader thematic analysis of each of two sets of data (one with people in recovery, one with supporters), attending closely to and theoretically developing the concept of an "eating disorder recovery assemblage" that is comprised of both human and non-human forces that impact the manifestation of recovery in the lives of people impacted by eating distress.

Prior examples of feminist research seeking to attend to that which exceeds the discursive exists, offering paths through work that allows us to "think with theory" (Jackson \& Mazzei, 2013) and to make visible the ways in which research does not entail unearthing a singular "truth" (Fox \& Alldred, 2015; Fullagar, 2017, 2020; Holmes, 2016). New materialisms are multiple but are held together by a common emphasis on moving beyond humanist approaches to integrate analyses of things and flows that are involved in generating, deploying, and experiencing power (Braidotti, 2018; Lupton, 2019). They have been used, for example, to analyze and make space for and sense of multiple coinciding birthing experiences (Chadwick, 2018), digital health technologies (Lupton, 2019), recoveries from depression (Fullagar, 2020), smoking experiences (Dennis, 2018), diagnostic experiences in relation to "Autism spectrum disorder" (Frigerio et al., 2018), puberty experiences (Rice, 2018), and more. Lupton (2019) 
compellingly describes the potential for new materialist social science research, highlighting how by focusing on the micropolitical, we are able to engage with complexities of embodied experiences in the world.

\section{Participants}

The study received ethics clearance through the University of Guelph ethics board (REB\#16-12-277). Twenty participants in recovery? (hereafter referred to as PIRs) were recruited using social media postings through the first author's account and circulated by local and national ED organizations. In keeping with our intention to understand individuals' experiences of 'eating disorders' and 'recovery' within and outside treatment regimes, we did not require diagnoses for participation, and recovery was described as "doing significantly better than during a time of acute distress" in order to avoid foreclosing the use of the term "recovery." Upon completion of an interview (ranging from approximately 30 minutes to approximately an hour and a half) each PIR was asked to select a supporter to be interviewed. PIRs largely identified as women (one identified as gender fluid). Fifteen identified as white, one as Greek, and four as Asian. Not all PIRs had been formally diagnosed with eating disorders or received treatment. Not all PIRs were able to identify a person who had meaningfully supported them in their recoveries. Anon 1 interviewed 14 supporters (hereafter referred to as

\footnotetext{
${ }^{2}$ Recovery is a fraught term; for the purposes of this study, participants self-selected into the study if they identified as "doing significantly better than they had been during a time of acute distress" and asked to reflect on their relationships to the term recovery (article to be published elsewhere on the specifics of these relationships).
} 
SPs): six partners (all identified as men), one father, one friend, two sisters, and four mothers. Of these, 11 identified as white, two as Greek, and one as Chinese.

All participants were informed about the study purpose (to understand experiences of recovery, including relationships to that concept, in relation to supporters and systems) and provided informed consent. PIRs were asked questions about their experiences of eating distress and "recovery" and their relationships to the way recovery is represented. SPs were interviewed about the role(s) they played in supporting a loved one during the distress, what they found or would have found helpful during this time, and their orientation to recovery as a concept and for their loved one.

The arrangement of relationships between PIRs and SPs challenges some commonly held assumptions about who might be most supportive in the eating disorder care assemblage. There is a primary focus on nuclear, heterosexual, white, middle class, two-parent families (and prototypically mothers) as primary supporters for people (typically daughters) with eating disorders, with a secondary emphasis on (often heteronormative) partners in the literature (e.g. Bulik et al. 2011). This focus may inadvertently reinforce dominant stereotypes about who eating disorders impact: that is, thin, young, white, heterosexual, middle to upper class women, whose families (and especially mothers) are able to support them full time. Further, by focusing on supporters including but not limited to legal or blood relatives, this work extends beyond classical mother-responsibilizing (and blaming) discourses so common in eating disorders rhetoric (McNab \& Kavner, 2001). 


\section{Analyses}

The analyses we present here foreground the co-creation of supportive assemblages of eating disorder recovery. What we find in this data is neither individual pursuit of recovery "against all odds" nor supporter altruism and self-denial. Instead, the facilitation of the eating disorder recovery assemblage comes about through affective, material, and discursive practices that present new possibilities for "being well." We describe supportive eating disorder recovery assemblages in relation to the mobilization of trust and love. These are mobilized within a particular sociocultural, historical, and relational moment in which the circulation of biopedagogical imperatives in society-as well as in eating disorder recovery spaces (LaMarre \& Rice, 2016, 2017)_impacts embodied experiences. In collectively creating supportive eating disorder recovery assemblages, PIRs and SPs navigated these biopedagogies differently; for PIRs, conflicting and contradictory biopedagogies issued in and outside of recovery-oriented spaces (i.e., treatment) impacted their orientation to SPs' or prospective supporters' actions. For SPs, supporting someone through the process of recovery brought them into confrontation —or at least consideration—with their own situatedness within biopedagogical imperatives. In what follows, we detail two key aspects of supportive eating disorder recovery assemblages as articulated by PIRs and SPs - trust and love. After exploring both in their discursive, material, and affective dimensions, we turn to an analysis of the biopedagogical inflections that mediate the creation of supportive eating disorder recovery assemblages. 


\section{Mobilizing Trust}

Many PIRs were used to not being trusted in and outside of treatment contexts; people with eating disorders are not always trusted to be authorities over their own accounts and practices (Boughtwood \& Halse, 2010; Lester, 2019; Malson et al., 2004; Saukko, 2008). Trust was generated in relationships that were deemed to be supportive by PIRs not through the statement "I trust you," but rather through continued and often quite subtle relational dynamics and practices.

The two extracts we present in this section demonstrate contrasting experiences of trust and surveillance mobilized in recovery relationships. Both are from PIRs. Trust is a sticky concept and might be evidenced not only through action (including speech acts) but also through space and non-action (including silence) or, perhaps nonjudgment that elicits particular affective responses from those within a relationship. For example, Cora (a PIR) shared:

I didn't feel judged by my mom. I could see that she was struggling with it, and that she didn't really know what to do, and she just wanted me to be happy, and...she did kind of feel guilty, I think, about it all. But throughout all of it, though, I didn't feel that judgement and because of that, that helped a lot. (Cora)

Here, Cora's mother did not demonstrate perfect understanding of Cora's embodied experiences and actions. This was made visible to Cora ("I could see that she was struggling with it, and that she didn't really know what to do") suggesting the existence of conflicting impulses. Cora did not specify how she "could see" that her mother was struggling; it is possible that this was made visible to Cora through both 
actions and affective resonance. She remarked upon how the desire for happiness and guilt comingled in the relationship she had with her mother throughout recovery ("she just wanted me to be happy, and... she did kind of feel guilty"). Despite these conflicts, Cora still described the relationship as supportive.

Braidotti (2015) articulates how affect can be mobilized as orientation to possible futures; arguably, Cora's mother mobilized trust and a lack of judgment and in so doing created the conditions of possibility for future-happiness. It is hard to nail down how this manifested; the "how" lies in the in-between of Cora's narrative. Cora went on to describe how for her, sometimes non-action (i.e. not being watched or told what to do) was more impactful than action. Cora's pathway to recovery was paved not through highly instructive methods but rather by having someone be there for and with her. This was evident throughout her interview; she described how her recovery was facilitated by being with people—but not necessarily people who knew that she was experiencing struggle. Later in the interview, she described how: "one of my things, my tricks I guess, to stay in recovery, was to always eat with people, never alone." In Cora's relationships, including with her mom, an over-focus on the eating disorder acted as an interrupting, rather than facilitating, force in the recovery assemblage. Safety was configured, here, in simply being with, rather than prescribing for or acting on.

The subtlety of trust in the supportive eating disorder recovery assemblage calls into question certain material practices often contrived to create "safety." Situations or configurations designed to be "safe" may not always be experienced as supportive or safe. Indeed, most PIRs identified relationships in which they had not been trusted or allowed space to enact recoveries in a way that aligned with their emerging preferred 
pace and practices. People with eating disorders are often assumed to be untrustworthy and eclipsed by the voices of their illness (see also Boughtwood \& Halse, 2010; Malson et al., 2011; Saukko, 2008). Particularly for PIRs who had been admitted to treatment many times, each subsequent admission made them subject to increased surveillance and distrust. This distrust could also be mobilized in post-treatment relationships, impacting the actions and re-actions of the person in relation to, for instance, family members.

As selfish as it might sound, actually protective of myself, too, because I don't want the police scene all over again and the badgering me and oftentimes even when I don't do as well or they notice that I'm struggling more, or I haven't finished a meal one day, I kind of get the cold shoulder from my family. I can tell that they're upset with me because I didn't eat what I should have eaten, or... and I know that it, that it's just frustration on their part, because they've lived with this for so long, but it makes me feel like their love and support is conditional for me. (Zoe, PIR)

This "police scene" of continued surveillance described here by Zoe was also tied to the bodily performance of recovery or disorder; when read as being "too thin" or not eating enough, participants experienced extreme skepticism and rejection from loved ones who knew of their histories of struggling around food, weight, and shape. Here we can observe a situation in which support was backgrounded or not enabled; other actors' skepticism, frustration, and upset crystallized conditions of impossibility of recovery and self-trust for participants like Zoe. Surveillance in recovery may be intended to create safety, but yet generate resistance and self-doubt. This is not to say 
that there is never a space for rules or watching in supporting recoveries, but rather that different actions, affects, and orientations may be variously helpful in different moments and in different ways throughout recoveries.

\section{Mobilizing Love}

Enacting a trusting space is not uncomplicated, nor is it about SP self-denial or self-subjugation. Both extracts in this section are from SPs. The first extract in this section presents an SP's perspective on "what it takes" to activate and mobilize a kind of radical trust. SPs described the love that they felt toward PIRs as a key aspect of their ability to support loved ones despite limited support for themselves and in spite of challenges. While it is beyond the scope of this paper to unpack the meaning of love, we might productively explore the mobilization of love in its complexity and potential. Love and overwhelm were sometimes described as lying hand in hand for supporters, generating the possibility of carrying on and creating supportive eating disorder recovery assemblages while simultaneously leading to exhaustion. A quote from SP Jeremy exemplifies affective exhaustion inflected by love:

I care about her. I mean, that's number one, I love her, she's my wife. And, you never want to see somebody that you love suffer [...] I don't necessarily have a short fuse, but if you keep hitting me with the same thing over and over again, I start to get frustrated, or irritated, and I feel guilty about that every time it happens. [...] When things are hard, when she's struggling with something, it's hard not say, "oh, this again?" But you need to filter that out, and be there for her, and at the same time find a way to let that go, whether you have to vent it 
through, my favourite is video games, but if we have something that we're dealing with and we get frustrated with each other, we'll eventually resolve the conflict, but in the meantime I go and play [video game] and shoot people in the face for a half hour. And they're sprites, and nobody gets hurt, and I'm happy. But yes, a little vent. I'd say, as a support, I need to find the time and space to find those vents without carrying it over and taking it out on her, because it's just a double whammy. (Jeremy)

This quote exemplifies the complexity of loving and supporting someone in recovery: it is not about being able to un-self-consciously care for another person without hesitation, at all times. In Jeremy's words, there is an intensity of feeling related to being unable to solve the eating disorder, which repeatedly surfaces. Love, here, is mobilized as primary emotion, but one that is coupled with the affective resonances of guilt and anger. Providing support simultaneously exhausts him and provides space for imperfection in recovery.

The co-presence of exhaustion, guilt, and frustration operates within a context of deep love and care; these emotions and embodied sensations might be interpreted as side effects of the deeply relational experiences of loving someone and seeing them suffer. As Jeremy noted, "you never want to see someone you love suffer." There is a strong impact of observing this suffering for long stretches of time; this weighed heavily on SPs and impacted their own state of mental wellbeing, particularly when SPs were unable to access support themselves. These rippling impacts of the eating disorder, in its relationality, stretched out into recovery. Some articulated the continued emotional impacts in clinical terms, for instance claiming the label of post-traumatic stress. 
Working within systems and seeking to help a loved one recover "at any cost" often, left supporters feeling like they should have taken greater care of their own emotional needs; as exemplified earlier, supporters often pushed these aside in favour of enacting care and support for a loved one.

We have sucked the life out of our health care system. I'm telling you right now. We sucked the life out of them. And you know what? I honestly can say is, I mean, it's hindsight, right? But, from a parent perspective, we didn't have time to do our own self-care. I should have been seeing someone. I was too busy seeing doctors and psychiatrists and stuff with my daughter, because it was familybased treatment. And don't get me wrong, I'm overjoyed that my daughter's doing well, and she's alive, but the thing is, is at what price? (Elizabeth, SP)

This excerpt further demonstrates the complexity of exploring the residue of emotions of guilt and exhaustion. Rather than identifying the cause of the continued ramifications of the carework as systemic, Elizabeth noted how she "should have been seeing someone" without explicitly identifying the reason why she was not seeing anyone. She did identify that she was busy, engaged with systems of care; however, she situated the blame within herself for not engaging in proper "self-care." She also explored the impact of her not having cared for herself in relation to her daughter's experience of her (Elizabeth's) continued suffering. We might also consider, once again, the self-responsibilization of the "caring mother," wherein Elizabeth reflects on the imperative to care for oneself (invoking "should"), even during times of stress and strain. There are shades of the expectations placed on supporters to navigate the twin currents 
of trust and surveillance here; arguably, supporters are stuck at the interstice of systems encouraging control and relational flows calling for trust and openness.

\section{Becoming Attuned}

Eating disorder recovery assemblages are not generated outside of broader social context, including discourses, affects, and instructions on how to live. Instone (2014) describes attunement as "learning [that] occurs in collective and mutually constituted relations, [as] worlds are re/made through engagement and encounter[s] across all manner of difference" (p. 80). The ways in which biopedagogies shaded supportive eating disorder recovery assemblages reveals the entangled nature of bodies, relationships, and contexts. The extracts in this section exemplify the tenor of biopedagogies through the supportive eating disorder recovery assemblage, through perspectives of both PIRs and SPs.

As noted above, it was important for PIRs to have people in their lives who created spaces for them to be at ease in their own versions of recovery-in fact, several noted that they often avoided disclosing their eating disorder past to others because they did not know what kinds of spaces others would make for their versions of recovery. PIRs' accounts demonstrate how their recoveries—and what they found supportive in recovery—was impacted by biopedagogical imperatives. As Robyn (a PIR) described:

... people stigmatize you. They make assumptions, and they expect certain things, and then they watch you. They watch you, what you're eating, and they judge. And I don't like that. So for me the decision to tell someone is always a 
really big deal and based on: is this gonna change the way that we interact? Are you suddenly going to start interacting with me weirdly, and is food, if we go out for a meal, is that gonna become weird? (Robyn)

As Robyn noted, comfort or ease is relational, particularly within an alreadysurveillant world; the biopedagogies for health circulating broadly intersect with those expected of people in recovery to generate a tight nexus to be navigated.

For SPs, supporting someone through recovery involved making space for fluctuating, not always conventional, and individual ways of embodying and living recovery. SPs lived, in some ways, at the threshold of the conflicting biopedagogical instructions for health (eating less, exercise more) issued in society more broadly and those that dictate what is "normal" (re-feeding and weight restoration) in eating disorder recovery. Sometimes this meant re-evaluating their own relationships with food, eating, and normalcy —-the biopedagogies dictating their own lives—in relation to their role as supporter, as Elliot (an SP) describes:

I think I have a relatively normal and healthy relationship with that $[\ldots]$ through struggling with [loved one] I am a bit more aware of making sure that I'm eating regularly and healthily and just remembering that, just trying to be positive about your body image... I've always had a pretty normal relationship with that but even after struggling with [loved one] l've come to be more aware of it as well (Elliot)

Elliot explains how "normalcy" around food and body is something that he had taken for granted before becoming a supporter; the affective intensity of the supporterPIR relationship brought into being a reflection on the nature of his own relationship with 
his body in order to make space for his loved one's recovery. Here, Elliot's conscious enactment of biopedogogies of "healthy" recovery might be interpreted as creating a contained, bounded or 'safe' space within the recovery dyad in which his partner could experiment with enacting her own version of recovery.

Some SPs' comments about the differences between "normative" (and, often, gendered) body shame they encountered and those of their loved ones also demonstrate the affective tenor of biopedagogies as they flow through eating disorder recovery assemblages and crystallize different variants of "normal." Alexis (an SP), for instance, reflected on her body history and the fact that she had not engaged in any extreme measures to alter her body:

I just go through the regular struggles that women go through, probably. Always wanting to lose a few pounds here or there, but never anything extreme. I've never been inherently unhappy with my body image, looking at my body, so I haven't done much to change it. [...] Or done anything extreme to change it, maybe. (Alexis)

As evidenced in this extract, there is a described difference between what "women" as a group might experience versus what someone with an eating disorder would do. In this excerpt in particular, the difference is described as being in the action, the intensity, and the degree to which it impacted the rest of life. For Alexis, the body image struggles she has experienced are normative insofar as they are not described as being debilitating or significantly interfering with the rest of her life. This also gives us clues to the ways in which eating disorders are framed by those who are not 
experiencing them, but who are seeing loved ones experience them. If the difference driving the extreme behaviour is the intensity of the feelings of body distress, then the supposed contributor to disordered behaviour is the centrality of body distress to selfconcept.

Supporters were aware that they would never fully be able to get into the headspace of PIRs, and commonly tried to listen to their loved ones as much as possible to learn from their experiences and provide tailored support that would meet their needs - particularly when this kind of support was significantly lacking outside of the relationship. As Scott (an SP), a partner, described:

I've always approached this in the same way, like there haven't been any insights that affected the way that I behaved towards her [...] living with someone who has an eating disorder, you naturally have more respect and understanding for people who have eating disorder, because it's not, as I said before, an isolated thing. Like there's a lot of other stuff and like the person cannot really help sit there doing, like they're going through with that type of behaviour, because the behaviour serves the purpose linked to a greater whole. (Scott)

In mobilizing respect and understanding, Scott contributed to generating an environment in which lived, embodied experience is valued—something not always found in treatment centres, where patriarchal (and parental as well as other modes of social) control may be exerted over the bodies and actions of those engaged in treatment (Gremillion, 2003). 


\section{Conclusion}

Our findings can be interpreted in a hopeful way, offering insight into perspectives on the roles that loved ones might play in supporting recoveries. We add attention to how supportive relationships are mobilized to feminist studies identifying the power and relational dynamics of eating disorders. This hopeful orientation is not possible to articulate without also acknowledging the affective exhaustion of creating space for recovery, or of recovering. It is important not to binarize relationships and support configurations into good or bad; the mobilization of trust and love and negotiation of support in the context of biopedagogies creates planes of possibility but not perfect solutions for recovery. PIRs and SPs continue to exist within, and in various relationships with, people and systems that constrain options for care and recovery for both PIRs and SPs, including biopedagogical imperatives. Exploring experiences of caring for a loved one and creating the spaces in which recoveries are made possible provides meaningful ways for supporters to become engaged with recoveries.

This work evinces the ways that people impact each other while they negotiate food, body management, and recovery in a biopedagogical world. As Lester (2019) writes, "eating disorders do not exist within people; they emerge between people" (p. 9). Our data speaks to the ways in which recovery, too, is tied up in the affective and intercorporeal. Together, PIRs and SPs negotiated complex terrains of recovery within a context that holds up specific dictates for both health and recovery. The importance of having space in which a variety of emotions were welcome and where exploration was 
made possible was key for many PIRs. Meanwhile, SPs, largely lived at the intersection between biopedagogies of caring and recovery and dominant health dictates.

Eating disorders are not only tied up in individual behaviours, attitudes, patterns, or cognitions, but rather in social flows and affects (Lester, 2019). Exploring affect helps us to understand "how interpersonal connections are forged, mediated, and undone in and through [affective] intensities" (Lester, 2019, p. 68). Eating disorder treatment can be configured as affective, making possible certain ways of being ill and well at the expense of alternatives (Lester, 2019). Our work demonstrates that relationships outside of the clinical context can entwine with embodied experiences of recovering from an eating disorder in a world imbued with moralizing perspectives on food and exercise. Perhaps in the project of co-constituting improvisational space for recovery, those in recovery and supporters forge spaces that mediate what Lester $(2019$, p. 187) describes as "the flood of affect" encountered by many who seek to recover. Supporters, too, encounter new ways of being and doing in interaction and entanglement with people in recovery.

Thinking through eating disorder recovery assemblages requires different ways of doing research and orienting to participants. Many included supporters were not members of the nuclear family traditionally imagined to be those taking caring roles. Considering how both SPs and PIRs navigate biopedagogies represents an important step toward deconstructing boundaries between the "sick" and the "well" or the "normal" and "abnormal" of eating and recovery, exploring how the affective intensities of biopedagogical society congeal and crystallize around and through people who do not 
themselves (currently) experience disordered eating but who live in close relation to those who do.

Our work is limited by our relatively homogenous and small sample; further, as noted, not all participants were able to identify a supporter. These aspects limit the ability to generate a "new path" toward eating disorder recovery. However, illuminating such a path would run counter to the ethic of this work, grounded in an openness, rather than foreclosure, to different ways of recovering. As noted in the introductory section, we also found that the affective resonances we experienced in interviews faded in the transcribed (or even recorded) texts. Future research might specifically interrogate affective relations of care to continue and tease out the nuances of support and care into recovery, perhaps through visual or artistic media that allow for deeper engagement with the affective at the level of presented data.

Presently, providing "resources for recovery" often means engaging with systems that may or may not be open to not only different versions of recovery, but to different bodies, full stop (Duff, 2016). Truly imagining eating disorder recoveries as "assembled" means moving beyond elaborations of recovery that envision it as an individual pursuit or one that is done abstracted from dominant discourses and teachings. This work begins to open to ways of supporting people in recovery and understanding eating disorder recoveries as dynamic and situated in time and space. Trust and love are mobilized in the supportive relationships described by both those in recovery and supporters. These are situated within the biopedagogical realities faced by both people in recovery and supporters, who exist in a nexus of affective, instructional, and material flows. These complexities raise the importance of troubling ideas around what 
constitutes "safety" in eating disorder recovery relationships; this is not to say that surveillance and control are never necessary, but to open new terrain for exploring how "being supportive" may look or feel like something more and otherwise.

This work builds on feminist analyses that have revealed the ways that people with eating disorders become caught up in essentializing discourses about who and what they "are" (e.g., Malson et al., 2011; Malson et al., 2004). It works alongside analyses of how the control exerted in eating disorder treatment may constrain possibilities for recovery by, to a certain extent, replicating imperatives for selfmanagement present in eating disorders themselves (Gremillion, 2003). Explicitly implicating biopedagogies allows for a consideration of the ways in which dictates for health and wellness issued in the mainstream conflict with prescriptions for eating disorder recovery (LaMarre \& Rice, 2016). Attending to affect, further, enables an orientation that takes up discursive practices that construct and configure people in recovery, supporters, and recovery itself in different ways and allows us to understand how "affective intensities" can act "as key strategies through which the neoliberal subject becomes engaged in the task of its own self-governance" (Parker \& Pausé, 2019, p. 251).

Supportive eating disorder recovery assemblages are not always easy to imagine in a society that presents limited material options for treatment, for instance through foregrounding managed care frameworks and vying for efficiency (Lester, 2019). However, by surfacing and analysing affect in relation to recovery and care, we can begin to explore alternative and nuanced approaches to support and caring in recoveries. In doing this theorizing, we begin to move beyond the taken-for-granted and 
to focus on the in-between. In so doing, we begin, ourselves, to affectively attune to eating disorder recovery and its shades and (im)possibilities.

\section{References}

Ahmed, S. (2004). The cultural politics of emotion. New York, NY: Routledge.

Ahmed, S. (2008). The politics of good feeling. Australian Critical Race and Whiteness Studies Association, 4(1),1-18.

Bardone-Cone, A.M., Hunt, R.A., \& Watson, H.J. (2018). An overview of conceptualizations of eating disorder recovery, recent findings, and future directions. Current Psychiatry Reports, 20(9), article 79.

Bordo, S. (1993). Unbearable weight: Feminism, western culture, and the body. Berkeley, CA: University of California Press.

Braidotti, R. (2002). Metamorphoses: Towards a materialist theory of becoming. Cambridge: Polity Press.

Braidotti, R. (2015, 7 October) Thinking as a nomadic subject. Lecture, ICI Berlin. Retrieved from https://www.ici-berlin.org/event/620/

Braidotti, R. (2018). A theoretical framework for the critical posthumanities. Theory, Culture \& Society, 36(6), 31-61.

Braun, V., \& Clarke, V. (2006). Using thematic analysis in psychology. Qualitative Research in Psychology, 3(2), 77-101.

Braun, V., \& Clarke, V. (2019). Reflecting on reflexive thematic analysis. Qualitative Research in Sport, Exercise, and Health, 11(4), 589-597.

Boughtwood, D., \& Halse, C. (2010). Other than obedient: Girls' constructions of doctors and treatment regimes for anorexia nervosa. Journal of Community and Applied Social Psychology, 20(2), 83-94. 
Bulik, C., Baucom, D., Kirby, J., \& Pietsky, E. (2011). Uniting Couples (in the treatment of Anorexia Nervosa (UCAN). International Journal of Eating Disorders, 44(1), 19-28.

Burrows, L. (2009). Pedagogizing families through obesity discourse. In J. Wright, \& V. Harwood (Eds.). Biopolitics and the 'obesity epidemic': Governing bodies (pp. 127-140). New York, NY: Routledge.

Cappellini, B., Harman, V., \& Parsons, E. (2018). Unpacking the lunchbox: Biopedagogies, mothering, and social class. Sociology of Health \& IIIness, 40(7), $1-15$.

Chadwick, R. (2018). Bodies that birth: Vitalizing birth politics. New York, NY: Routledge.

Cliff, K., \& Wright, J. (2010). Confusing and contradictory: Considering obesity discourse and eating disorders as they shape body pedagogies in HPE. Sport, Education and Society, 15(2), 221-233.

Clough, P.T. (2003). Affect and control: rethinking the body 'beyond sex and gender'. Feminist Theory, 4(3), 359-364.

Deleuze, G., \& Guattari, F. (1987). Becoming-intense, becoming-animal, becomingimperceptible In G. Deleuze \& F. Guattari, A thousand plateaus: Capitalism and schizophrenia (pp. 232-309). London, MN: University of Minnesota Press.

Dennis, S. (2018). Becoming enwinded: A new materialist take on smoking pleasure. International Journal of Drug Policy, 51, 69-74.

de Vos, J.A., LaMarre, A., Radstaak, M., Bijkerk, C.A., Bohlmeijer, E.T., \& Westerhof, G.J. (2017). Identifying fundamental criteria for eating disorder recovery: A systematic review and qualitative meta-analysis. Journal of Eating Disorders, 5, article 34.

Duff, C. (2014). Assemblages of health: Deleuze's empiricism and the ethology of life. Rotterdam, NL: Springer International. 
Duff, C. (2016). Atmospheres of recovery: Assemblages of health. Environment and Planning, 48(1), 58-74.

Eli, K. (2014). Between difference and belonging: Configuring self and other in inpatient treatment for eating disorders. PLoS One, 9(9), e105452.

Frigerio, A., Benozzo, A., Holmes, R., \& Runswick-Cole, K. (2018). The doing and undoing of the "autistic child": Cutting together and apart interview-based empirical materials. Qualitative Inquiry, 24(6), 390-402.

Foucault, M. (1988). Technologies of the self: $A$ seminar with Michel Foucault. L.H. Martin, H. Gutman \& P.K. Hutton (Eds) Boston, MA: University of Massachusetts Press.

Fox, N.J., \& Alldred, P. (2015). New materialist social inquiry: designs, methods and the research-assemblage. International Journal of Social Research Methodology, 18(4), 399-414.

Fullagar, S. (2017). Post-qualitative inquiry and the new materialist turn: Implications for sport, health and physical culture research. Qualitative Research in Sport, Exercise and Health, 9(2), 247-257.

Fullagar, S (2020). Diffracting mind-body relations: Feminist materialism and the entanglement of physical culture in women's recovery from depression. In $\mathrm{J}$. Newman, H. Thorpe, \& D. Andrews (Eds.), Moving body: Sporting ecologies, assemblages, and new materialism. Durham, NC: Duke University Press.

Gregg, M., \& Seigworth, G.J. (2010). The affect theory reader. Durham, NC: Duke University Press.

Gremillion, H. (2003). Feeding anorexia: gender and power at a treatment center. Durham and London: Duke University Press.

Holmes, S. (2016). 'Blindness to the obvious'? Treatment experiences and feminist approaches to eating disorders. Feminism \& Psychology, 26(4), 464-486.

Instone, L. (2014). Unruly grasses: Affective attunements in the ecological restoration of urban native grasslands in Australia. Emotion, Space, and Society, 10, 79-86. 
Jackson, A.Y., \& Mazzei, L.A. (2013). Plugging one text into another: Thinking with theory in qualitative research. Qualitative Inquiry, 19(4), 261-271.

Jenkins, J., \& Ogden, J. (2012). Becoming 'whole' again: A qualitative study of women's views of recovering from anorexia nervosa. European Eating Disorders Review, 20, e23-e31.

LaMarre, A., \& Rice, C. (2016). Normal eating is counter-cultural: Embodied experiences of eating disorder recovery. Journal of Community and Applied Social Psychology, 26(2), 136-149.

LaMarre, A., \& Rice, C. (2017). Hashtag Recovery: \#Eating Disorder Recovery on Instagram. Social Sciences, 6(3), article 68.

LaMarre, A., Rice, C., \& Jankowski, G. (2017). Eating disorder prevention as biopedagogy. Fat Studies, 6(3), 241-254.

Lavis, A. (2015). Careful starving: Exploring (not) eating, care and anorexia. In E-J. Abbots, A. Lavis, \& L. Attala (Eds.) Careful eating: Bodies, food and care. Farnham, UK: Ashgate.

Lester, R.J. (2018). Ground zero: Ontology, recognition, and the elusiveness of care in American eating disorders treatment. Transcultural Psychiatry, 55(4), 516-533.

Lester, R.J. (2019). Famished: Eating disorders and failed care in America. Oakland, CA: University of California Press.

Linville, D., Brown, T., Sturm, K. \& McDougal, T. (2012). Eating disorders and social support: Perspectives of recovered individuals. Eating Disorders: The Journal of Treatment and Prevention, 20(3), 216-231.

Lupton, D. (2019). Toward a more-than-human analysis of digital health: Inspirations from feminist new materialism. Qualitative Health Research, 29(14), 1998-2009.

MacNeill, M., \& Rail, G. (2010). The visions, voices and moves of young 'Canadians': Exploring diversity, subjectivity and cultural constructions of fitness and health. In J. Wright, \& D. Macdonald (Eds.), Young people, physical activity and the everyday (pp. 175-193). London, UK: Routledge. 
Malson, H. (1998). The thin woman: Feminism, post-structuralism and the social psychology of anorexia nervosa. New York, NY: Routledge.

Malson, H., Finn, D.M., Treasure, J., Clarke, S., \& Anderson, G. (2004). Constructing "the eating disordered patient": A discourse analysis of accounts of treatment experiences. Journal of Community and Applied Social Psychology, 14(6), 473489.

Malson, H., \& Burns, M. (2009). Re-theorising the slash of dis/order: an introduction to critical feminist approaches to eating dis/orders. In H. Malson, \& M. Burns (Eds.), Critical feminist approaches to eating dis/orders (pp. 1-5). New York: Routledge.

Malson, H., Bailey, L., Clarke, S., Treasure, J., Anderson, G., \& Kohn, M. (2011). Un/imaginable future selves: A discourse analysis of in-patients' talk about recovery from an "eating disorder". European Eating Disorders Review, 19(1), 25-36.

Massumi, B. (2015). The politics of affect. Cambridge, UK: Polity Press.

Matoff, M.L., \& Matoff, S.A. (2001). Eating disorder recovery: Learning from the client's healing journey. Women \& Therapy, 23(4), 43-54.

McNab, S., \& Knaver, E. (2001). When it all goes wrong - challenge to mother blame: forging connections between mother and daughter. Journal of Family Therapy, 23(2), 189-207.

Parker, G., \& Pausé, C. (2019). Productive but not constructive: The work of shame in the affective governance of fat Pregnancy. Feminism \& Psychology, 29(2), 250268.

Piran, N. (2004). Teachers: on "being" (rather than "doing") prevention. Eating Disorders, 12(1), 1-9.

Price-Robertson, R., Manderson, L., \& Duff, C. (2017). Mental ill health, recovery and the family assemblage. Cultural Medicine and Psychiatry, 41(3), 407-430. 
Rail, G., \& Jette, S. (2015). Reflections on biopedagogies and/of public health: On bioothers, rescue missions, and social justice. Cultural Studies $\leftrightarrow$ Critical Methodologies, 15(5), 327-336.

Rice, C. (2009). Imagining the other? Ethical challenges of researching and writing women's embodied lives. Feminism \& Psychology, 19(2): 245-266.

Rice, C. (2014). Becoming women: The embodied self in image culture. Toronto, ON: University of Toronto Press.

Rice, C. (2015). Rethinking fat: From bio- to body-becoming pedagogies. Cultural Studies $\leftrightarrow$ Critical Methodologies, 15(5), 387-397.

Rice, C. (2018). The spectacle of the child woman: Troubling girls and the science of early puberty. Feminist Studies, 44(3). pp. 535-566.

Rice, C. (2018). Volatile bodies and vulnerable researchers: The risks of embodiment research. In S. Batacharya \& R. Wong, (Eds.) Sharing Breath: Embodiment, Pedagogy and Decolonization. (pp. 135-160). Edmonton: AU Press.

Rinaldi, J., LaMarre, A. \& Rice, C. (2016). Recovering bodies: The production of the recoverable subject in eating disorder treatment regimes. In J. Coffey, S., Budgeon, and H. Cahill (Eds.). Learning Bodies - The body in youth and childhood studies (pp. 157-172). New York: Springer.

Rodin, J., Silberstein, L. R., \& Striegel-Moore, R. H. (1984). Women and weight: A normative discontent. In T.B. Sonderegger (Ed.), Nebraska symposium on motivation: Psychology and gender (pp. 267-307). Lincoln, NE: University of Nebraska Press.

Romano, K.A. \& Ebener, D. (2019). Disparities in psychological well-being based on subjective and objective eating disorder recovery statuses, and recovery status concordance. Eating Disorders: The Journal of Treatment \& Prevention, 27(1), 82-99.

Saukko, P. (2008). The anorexic self: A personal, political analysis of a diagnostic discourse. New York, NY: SUNY Press. 
Slof-Op't Landt, M. C., Dingemans, A. E., de la Torre Y Rivas, J., \& van Furth, E. F. (2019). Self-assessment of eating disorder recovery: Absence of eating disorder psychopathology is not essential. International Journal of Eating Disorders, 52(8), 956-961.

Stephens, E. (2015). Bad feelings: An affective genealogy of feminism. Australian Feminist Studies, 30(85), 273-282.

Tantleff-Dunn, S., Barnes, R.D., \& Larose, J.G. (2011). It's not just a 'woman thing': The current state of normative discontent. Eating Disorders, 19(5), 392-402.

Wetherall, M. (2012). Affect and emotion: A new social science understanding. Thousand Oaks, CA: Sage Publishing.

Whitney, J., Haigh, R., Weinman, J., \& Treasure, J. (2007). Caring for people with eating disorders: factors associated with psychological distress and negative caregiving appraisals in carers of people with eating disorders. British Journal of Clinical Psychology, 46(4), 413-28.

Wright, J., \& Halse, C. (2014). The healthy child citizen: Biopedagogies and web-based health promotion. British Journal of Sociology of Education, 35(6), 837-855.

Wright, J., \& Harwood, V. (Eds.)(2009). Biopolitics and the 'obesity epidemic': Governing bodies. New York, NY: Routledge.

\section{Biographical Note}

Andrea LaMarre, $\mathrm{PhD}$, is a lecturer in critical health psychology at Massey University in New Zealand. Her work has primarily focused on eating disorder recovery from a critical feminist perspective. She uses qualitative and arts-based approaches to explore recoveries in their sociocultural contexts and as they are embodied and embedded in relationships. 
Dr. Carla Rice is Professor and Canada Research Chair at the University of Guelph and Academic Director of the Re $\bullet$ Vision Centre for Art and Social Justice, a research creation centre with a mandate to use arts-informed methods to foster wellbeing, equity, and justice. Her research program investigates the power of art and story to re-imagine the human within and across disciplines and systems, including education, healthcare, and the arts. 